\title{
Editorial
}

\section{Rehabilitation Robotics 2013}

\section{Kazuhiko Terashima, ${ }^{1}$ Shigeyuki Suzuki, ${ }^{2}$ Oliver Sawodny, ${ }^{3}$ Ryojyun Ikeura, ${ }^{4}$ Ken'ichi Yano, ${ }^{5}$ and Ryo Saegusa ${ }^{6}$}

${ }^{1}$ Department of Mechanical Engineering, Center of Human and Robotics Symbiosis Research, Toyohashi University of Technology, Hibarigaoka 1-1, Tempaku-cho, Toyohashi 441-8580, Japan

${ }^{2}$ Graduate School of Medicine, Rehabilitation, Physicotherapeutics, Nagoya University, Taikou Minami 1-1-2, Higashi-ku, Nagoya 461-8673, Japan

${ }^{3}$ Leiter Institut für Systemdynamik, Universität Stuttgart, Pfaffenwaldring 9, 70569 Stuttgart, Germany

${ }^{4}$ Division of Mechanical Engineering, Graduate School of Engineering, Mie University, Kurimamachiya 1577, Tsu 514-8507, Japan

${ }^{5}$ Department of Mechanical Engineering, Faculty of Engineering, Mie University, 1577 Kurimamachiya-cho, Tsu 514-8507, Japan

${ }^{6}$ Center for Human-Robot Symbiosis Research, Toyohashi University of Technology, 1-1 Hibarigaoka, Tempaku, Toyohashi, Aichi 441-8580, Japan

Correspondence should be addressed to Kazuhiko Terashima; terasima@me.tut.ac.jp

Received 8 January 2014; Accepted 8 January 2014; Published 18 March 2014

Copyright (c) 2014 Kazuhiko Terashima et al. This is an open access article distributed under the Creative Commons Attribution License, which permits unrestricted use, distribution, and reproduction in any medium, provided the original work is properly cited.

The current world has problems with the declining birth rate and aging population. With this problem, application of robotics technology to commodity life is highly required. Especially, demand of rehabilitation robotics becomes more and more increasing. Hence, a special issue on rehabilitation robotics introducing the forefront rehabilitation assist technology is planned in this journal.

The paper by G. Belforte et al., "Bra.Di.P.O. and P.I.G.R.O.: innovative devices for motor learning programs" presents two mechatronics prototypes, useful for robotic neurotreatments and new clinical trainings. P.I.G.R.O. (pneumatic interactive gait rehabilitation orthosis) is an active exoskeleton with an electropneumatic control. It imposes movements on lower limbs in order to produce in the patient's brain proper motor cortex activation. Bra.Di.P.O. (brain discovery pneumatic orthosis) is an MR-compatible device, designed to improve fMRI (functional magnetic resonance imaging) analysis. The two devices are presented together because both are involved in the study of new robotic treatments of patients affected by ictus or brain stroke or in some motor learning experimental investigations carried on healthy subjects.
The paper "Swarm robot control for human services and moving rehabilitation by sensor fusion" by T. Dewi et al. concentrates on swarm service robots. This study applies sensor fusion and swarm concept for service mobile robots in human services and rehabilitation environment. The swarm robots follow the human moving trajectory to provide support to human moving and perform several tasks required in their living environment. This study applies a reference control and proportional-integral (PI) control for the obstacle avoidance function.

The paper "A bio-inspired 10 DOF wearable powered arm exoskeleton for rehabilitation" by S. K. Manna et al., S. Bhaumik develops exoskeleton device (Exorn) comprised of ten degrees of freedom to control joints starting from shoulder griddle to wrist to provide better redundancy, portability, and flexibility to the human arm motion. A 3D conceptual model is being designed to make the system wearable by human arm. All the joints are simple revolute joints with desired motion limit. A Simulink model of the human arm is being developed with proper mass, length, and mass moment of inertia to determine proper torque required to actuate those joints. 
The paper "Constraint study for a hand exoskeleton: human hand kinematics and dynamics" by F. Chen Chen et al. provides a complete summary of the kinematic and dynamic characteristics of the human hand as a preliminary step towards the development of hand devices such as prosthetic/robotic hands and exoskeletons imitating the human hand shape and functionality.

The paper "Exoskeleton technology in rehabilitation: towards an Emg-based orthosis system for upper limb neuromotor rehabilitation" by L. M. Vaca Benitez et al. gives an overview on different kinds of neuromuscular deseases, review different forms of therapy and explain possible fields of rehabilitation and benefits of robotic aided rehabilitation. Next the mechanical design and control scheme of an upper limb orthosis for rehabilitation are presented.

The paper "Motion path design for specific muscle training using neural network" By K. Itokazu et al., proposes algorithms for designing a motion path capable of strengthening specific muscles. By using the proposed algorithms, it is possible to design a motion path maximizing the activity of an agonist muscle and minimizing that of other muscles. For training, the load is applied by using a 2-link arm. EMG signal is measured during a training experiment and the degree of muscular revitalization is evaluated by the amplitude of EMG signal.

The paper "Design and evaluation of the AIRGAIT exoskeleton: leg orthosis control for assistive gait rehabilitation" by M. A. Mat Dzahir and S. Yamamoto introduces the body weight support gait training system known as the AIRGAIT exoskeleton and delves into the design and evaluation of its leg orthosis control algorithm. This research proposes a simple paradigm for controlling the mono- and biarticular actuator movements cocontractively by introducing a cocontraction model.

The paper "An obstacle avoidance method for action support 7-DOF manipulators using impedance control" by M. Hamaguchi and T. Taniguchi proposes an obstacle avoidance method of action support 7-DOF manipulators. The manipulators are controlled with impedance control to follow user's motions. 7-DOF manipulators are able to avoid obstacles without changing the orbit of the end-effector because they have kinematic redundancy.

The paper "Development of assistive robots using international classification of functioning, disability, and healthconcept, applications, and issues" by $\mathrm{H}$. Tanaka et al. proposes a framework of evaluation and design of assistive robots using ICF (International Classification of Functioning, Disability, and Health). The goal of the framework is realization of the life design and the improvement of the quality of life using assistive technologies.

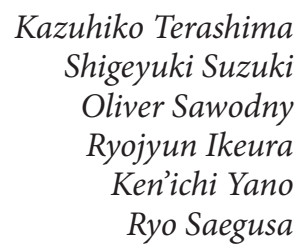



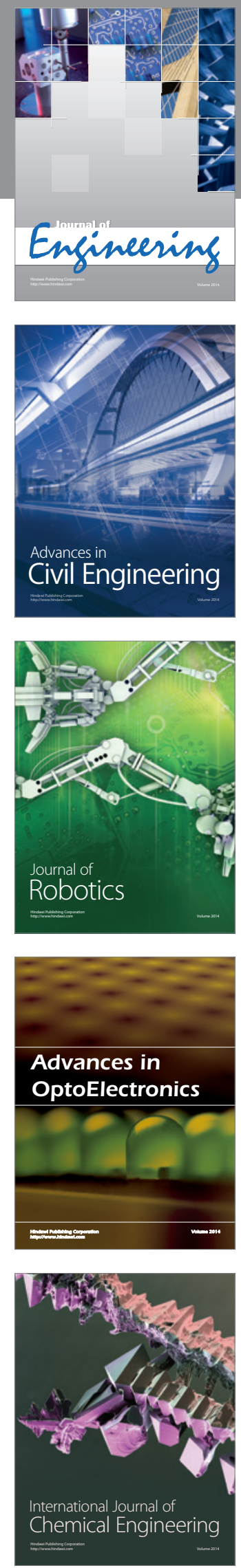

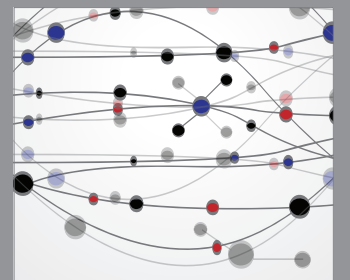

The Scientific World Journal
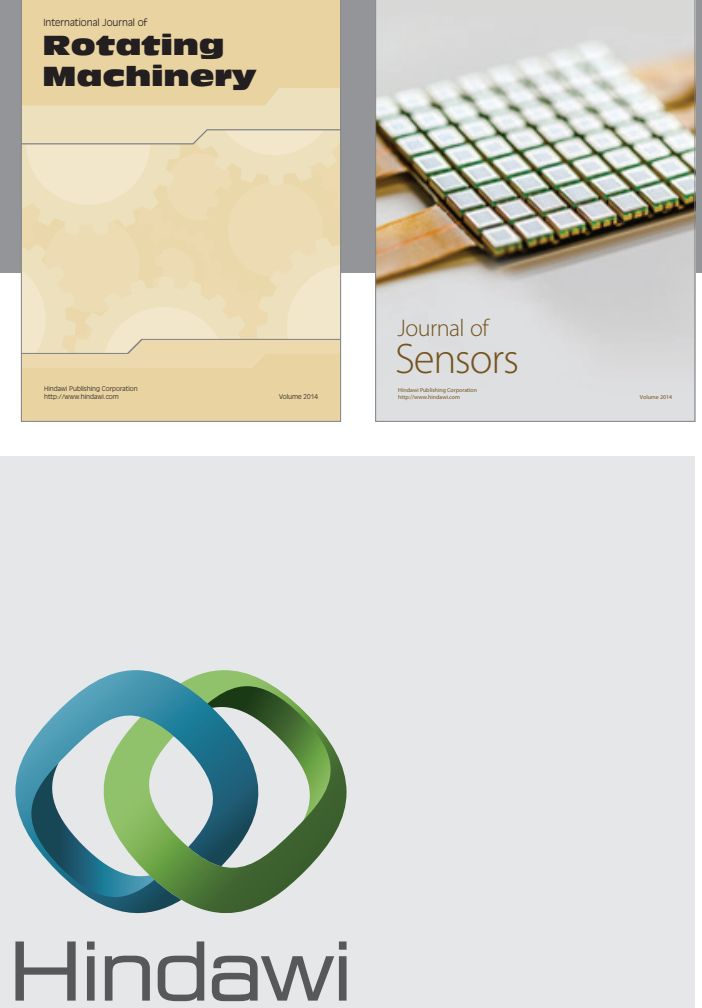

Submit your manuscripts at http://www.hindawi.com
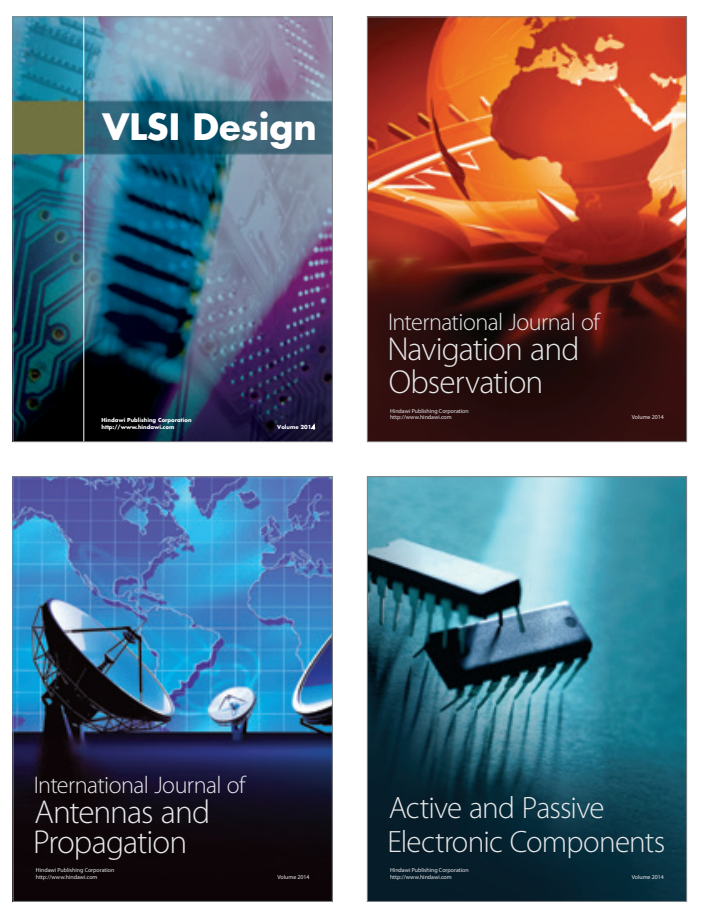
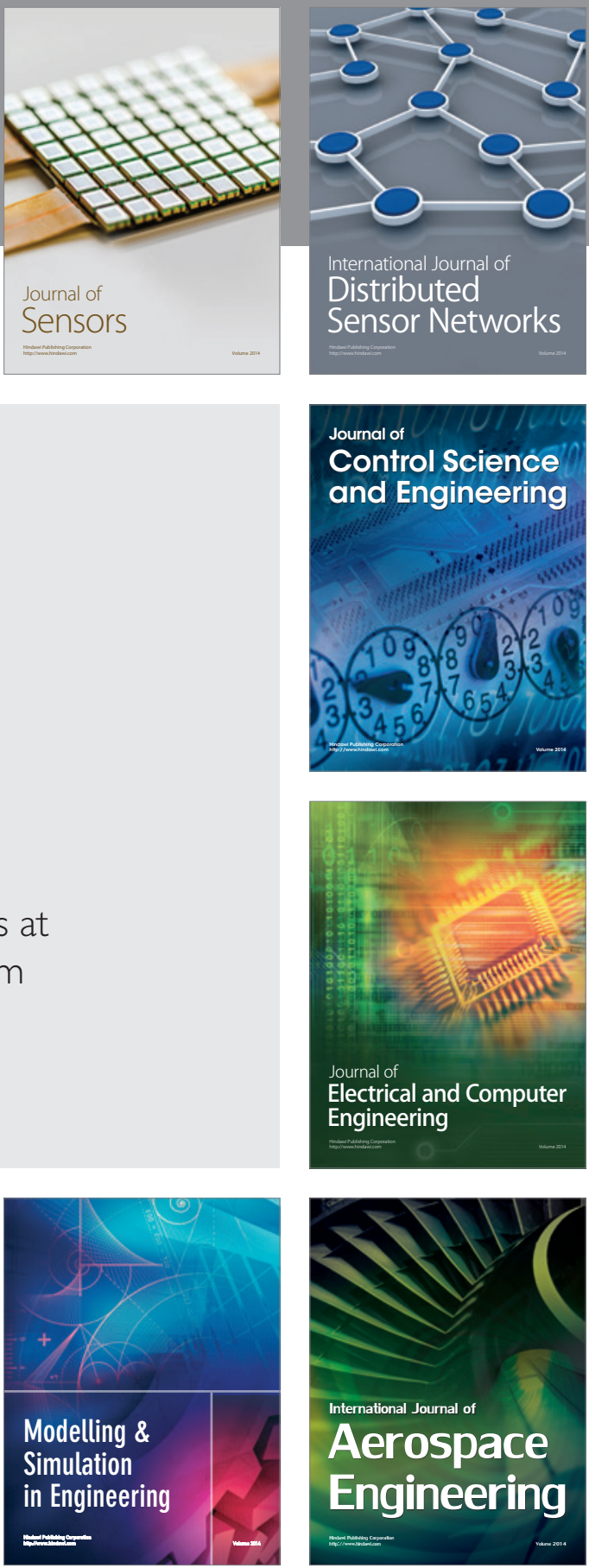

Journal of

Control Science

and Engineering
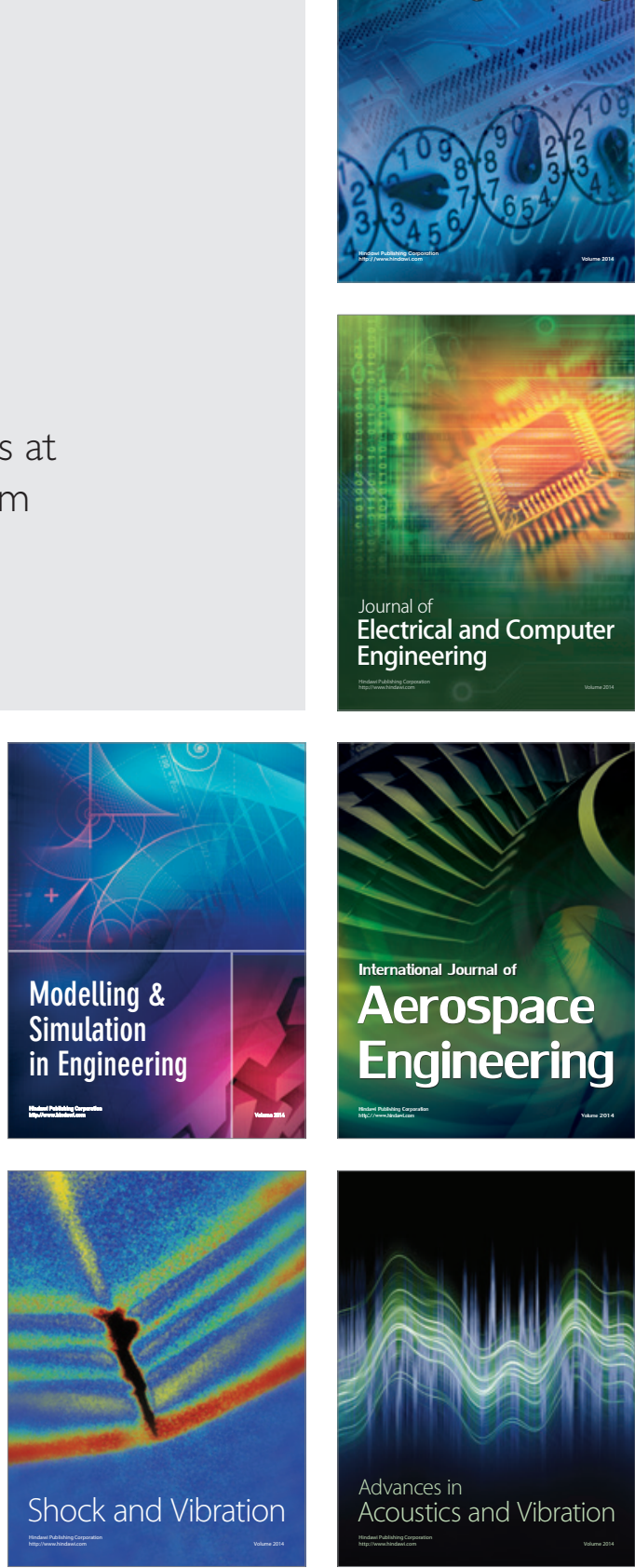